\title{
Aging Effect on Tapping Frequency Assessed by Hand Dominance
}

\author{
Yutaka Ueda $^{1}$, Satoshi Nishizawa ${ }^{2}$, and Tasuku Kimura ${ }^{1}$ \\ 1 Division of Anthropology, Graduate School of Science, The University of Tokyo, Tokyo \\ 2 Department of Kinesiology, Tokyo Metropolitan Institute of Gerontology, Tokyo
}

(Submitted April 23, 1999; Review sent May 21, 1999; Accepted October 18, 1999)

\begin{abstract}
Mean intertap intervals (MII) of each hand of 121 normal male and female adults and 12 female professional pianists were measured in order to clarify three effects on the maximum free tapping frequency, namely, dominance of the human hand, aging, and the impact of daily exercise. The maximum free finger tapping test can quantify motor agility in hands and fingers. The normal adult group consisted of 47 young urban university students and 74 elderly rural residents. Results of a three-way analysis of variance (ANOVA) of their MIIs revealed that all main effects were significant but interactions were not. Twelve professional female pianists (6 young and 6 elderly) had their MIIs analyzed together with those of normal females via three-way ANOVA. Results of the ANOVA showed that all main effects were significant and an interaction between expertise versus hand dominance was significant. Using Tukey's HSD, pianists were found to exhibit no differences between their hands. The results show that aging, gender and hand dominance all affect hand agility in normal people. Though pianists have equivalent and relatively high agility in both hands, aging diminishes the agility as equally as in normal people. These results imply that regular training of hands cannot offset the gradual decrease of manual dexterity that occurs with aging.
\end{abstract}

Keywords: maximum tapping frequency, Mean Intertap Intervals, aging effects, hand dominance, training 


\section{Introduction}

Precision manual movements and control are one of the unique motor characteristics of humans (Kapandji, 1974). Only humans have hands which are free from locomotor demands. Human hands can make highly sophisticated tools, manipulate complexed musical instruments and achieve numerous other kinds of daily tasks. Such coordinated manual dexterity is one of the most fundamental motor abilities in our daily and cultural lives (Fleishman and Quaintance, 1984). Difficulty with or loss of manual coordination seriously decreases our activity levels (Hashizume and others, 1994).

Tracking tests or reaction time tests have been the principal methods used to investigate manual coordination in the cognitive and psychological sciences. These studies, however, mainly focus on qualitative aspects of manual coordination. Among the few quantitative investigations of manual skill, Hashizume and others (1994), using the tapping method, found that manual speed deteriorates with age.

From an anthropological point of view, three physical factors influence manual coordination (Spirduso, 1995). First, age related changes can result in a generalized deterioration of motor speed. Various studies have shown a loss of coordination due to a slowing motor speed in many motor acts such as gait (Spirduso, 1995). Second, hand dominance creates difficulties when humans perform dual hand coordinated skills such as typewriting or piano playing. Third, the necessity of a learning and an exercising period is vital for the improvement and accomplishment of fully coordinated manual skills. Though the effects of these factors have been independently researched, quantitative relationships of these factors have not been studied.

In the present paper, we focus on the effects of aging, hand dominance and exercise on manual dexterity measured quantitatively by the tapping method. We also attempt to clarify the relationship among these three factors.

\section{Subjects and Methods}

\section{Subjects}

Subjects in this study were divided into two groups.

The first group, the "non-pianist group", was used for assessing the general effects of normal aging, hand dominance and gender. The group was subdivided into age (young or elderly) and gender (Table 1). Elderly participants were recruited from among residents of Wakuya-Town in Miyagi Prefecture, Japan. Young participants were university students from an urban community of Tokyo.

The second group, the "pianist group" was selected for determining the effects of aging, hand dominance, and their motor expertise due to continuous practice of hand use. Almost all members of this group had graduated first on the list of some music academy in Europe, won prizes in international competitions and performed as a professional concert pianist. Only female subjects could be recruited and they were subdivided with age (young or elderly) (Table 2).

We defined the subjects over 60 years old as elderly group. Pianist and non-pianist 
Table 1 Number and mean age of each category (gender and age) in the non-pianist group

\begin{tabular}{lcccccc}
\hline & \multicolumn{2}{c}{ young } & & \multicolumn{2}{c}{ elderly } \\
\cline { 2 - 3 } \cline { 6 - 7 } & $\mathrm{n}$ & Age & & $\mathrm{n}$ & Age \\
\hline male & 22 & $23.8 \pm 3.2$ & & 45 & $71.9 \pm 4.4$ \\
female & 25 & $22.9 \pm 3.2$ & & 29 & $71.1 \pm 3.1$ \\
\hline
\end{tabular}

Table 2 Number and mean age of each category in the pianst group

\begin{tabular}{ccccccc}
\hline & \multicolumn{2}{c}{ young } & & \multicolumn{2}{c}{ elderly } \\
\cline { 2 - 3 } \cline { 5 - 6 } & $\mathrm{n}$ & Age & & $\mathrm{n}$ & Age \\
\hline female & 6 & $29.9 \pm 3.5$ & & 6 & $65.2 \pm 3.3$ \\
\hline
\end{tabular}

groups contained a small difference in mean age of young and elderly subgroups.

No one in either group suffered from Parkinson's disease or had a history of cardiovascular disease.

The hand best able to manipulate chopsticks, scissors and a knife was defined as the dominant hand.

\section{Maximum finger tapping test}

We used the maximum finger tapping test in order to measure quantitatively the manual dexterity of each subject as defined by the maximum frequency of physiological coordination in the wrist and metaphalangeal joint.

The method of the finger tapping test has been described in detail by Nagasaki and

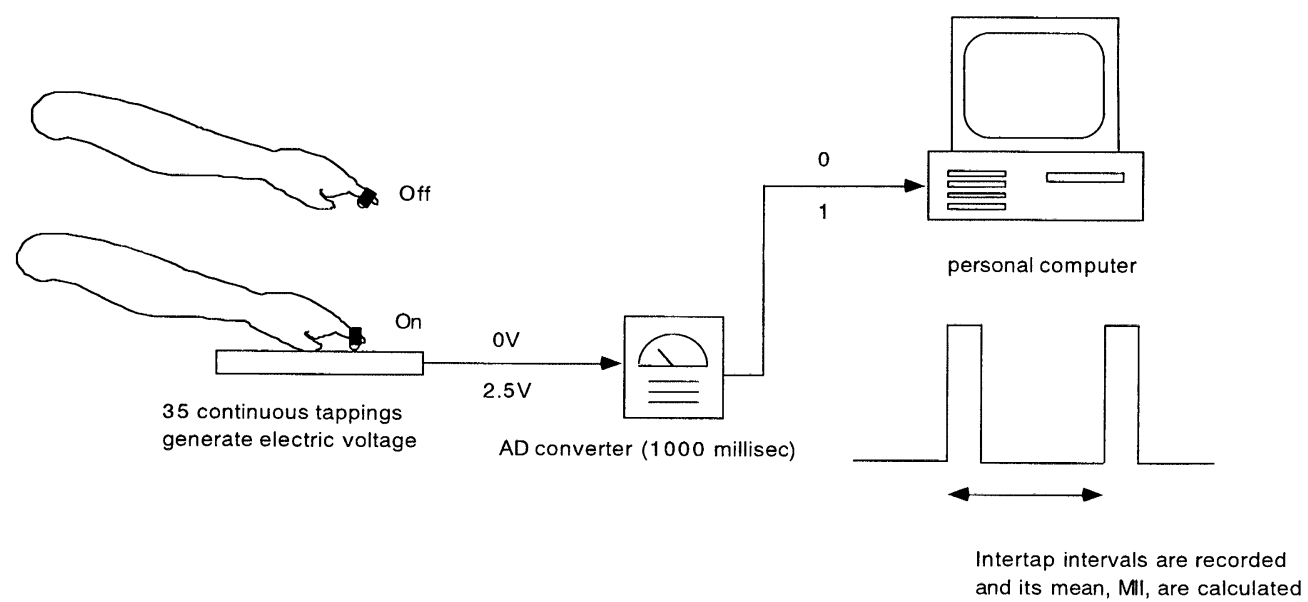

Figure 1 Diagram of the measuring system. 
others (1988). Subjects tapped on a stainless-steel plate using the tip of the middle finger to which a small metal knob was attached (Figure 1). The metal knob and the plate formed an electrical circuit which closed when the finger touched the plate, signaling the precise instant of the tap. Intertap intervals in each sequence of 35 tapping trials were measured on in milliseconds and processed on-line by an NEC PC-9800 computer. After a few seconds of exercise, subjects performed free tapping at their maximum rate. They first performed the task with their dominant hand, then with their non-dominant hand. The only instruction to subjects was not to allow their hands and forearms to tremor because of extreme haste.

\section{Analysis}

From intertap intervals of the 35 tapping trials, we calculated Mean Intertap Intervals (MII) and analyzed the MII as a parameter of manual agility.

To determine the effects of aging, hand dominance and gender on MII, we analyzed MII's of the non-pianist group with a 3-way analysis of variance. Gender (male or female) and age (young or elderly) were inter-subject factors. Hand dominance (dominant or non-dominant) was the intra-subject factor.

We also analyzed MII's of the pianist group and of females in the non-pianist group to assess the effects of aging, hand dominance and expertise. Expertise (pianist or non-pianist) and age (young or elderly) were inter-subject factors. Hand dominance was the intra-subject factor.

\section{Results}

Effects of aging, hand dominance, gender and their relationships in the non-pianist group (Table 3)

In the 3-way ANOVA, all the main effects were significant whereas no interactions were significant. As is evident from Figure 2, males were able to tap more frequently than females, young subjects tapped more frequently than elderly subjects, and the tapping frequency of the dominant hand was faster than that by the non-dominant hand.

Table 3 Results of 3-way ANOVA in the non-pianist group: There are significant main effects for the 3 factors but no significant interactions

\begin{tabular}{lcccc}
\hline & df of effects & df of error & F value & p value \\
\hline age & 1 & 118 & 174.9 & 0.001 \\
gender & 1 & 118 & 334.2 & 0.001 \\
dominance & 1 & 118 & 67.6 & 0.001 \\
age $\times$ gender & 1 & 118 & 0.42 & 0.52 \\
age $\times$ dominance & 1 & 118 & 1.10 & 0.30 \\
gender $\times$ dominance & 1 & 118 & 0.15 & 0.70 \\
age $\times$ gender $\times$ dominance & 1 & 118 & 0.17 & 0.68 \\
\hline
\end{tabular}




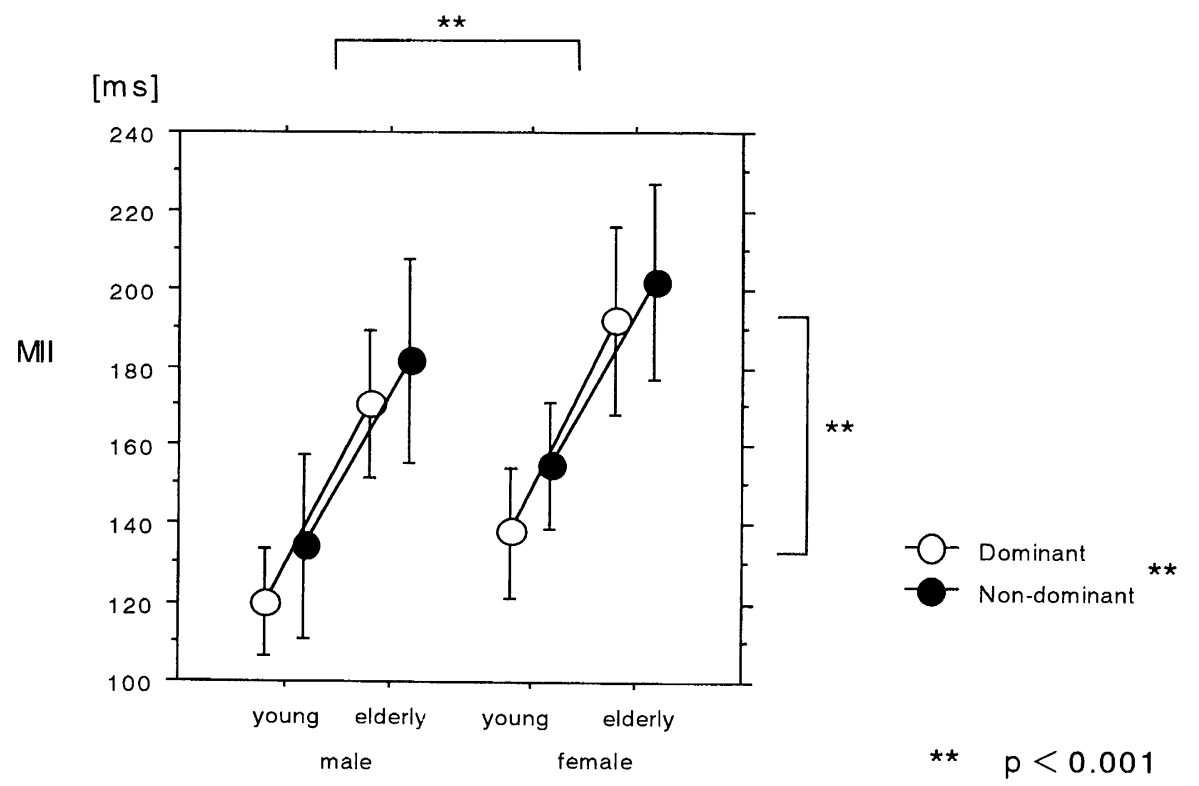

Figure 2 MII (Mean Intertap Interval) of the non-pianist group: The ordinate scale is MII in milliseconds. A higher MII means lower agility. The abscissa shows age and gender. White and black dots represent dominant and non-dominat hands, respectively. Vertical bars are \pm 1 SD of the MII.

Though the tapping frequency was greater in young group compared to the elderly, variation in an age group (approximately 50 milliseconds) were common in both genders and in both hands.

Effects of aging, hand dominance, expertise and relationships between pianists and non-pianists (Table 4)

Table 4 Results of a 3-way ANOVA for pianist and non-pianists groups: There are significant main effects for the 3 factors but a significant interaction occurs only between in expertise and hand dominance

\begin{tabular}{lcccc}
\hline & df of effects & df of error & F value & p value \\
\hline age & 1 & 60 & 44.6 & 0.001 \\
expertise & 1 & 60 & 36.6 & 0.001 \\
dominance & 1 & 60 & 12.5 & 0.001 \\
agexexpertise & 1 & 60 & 3.9 & 0.053 \\
agexdominance & 1 & 60 & 0.6 & 0.437 \\
expertisexdominance & 1 & 60 & 6.8 & 0.012 \\
agexexpertise $\times$ dominance & 1 & 60 & 0.1 & 0.795 \\
\hline
\end{tabular}




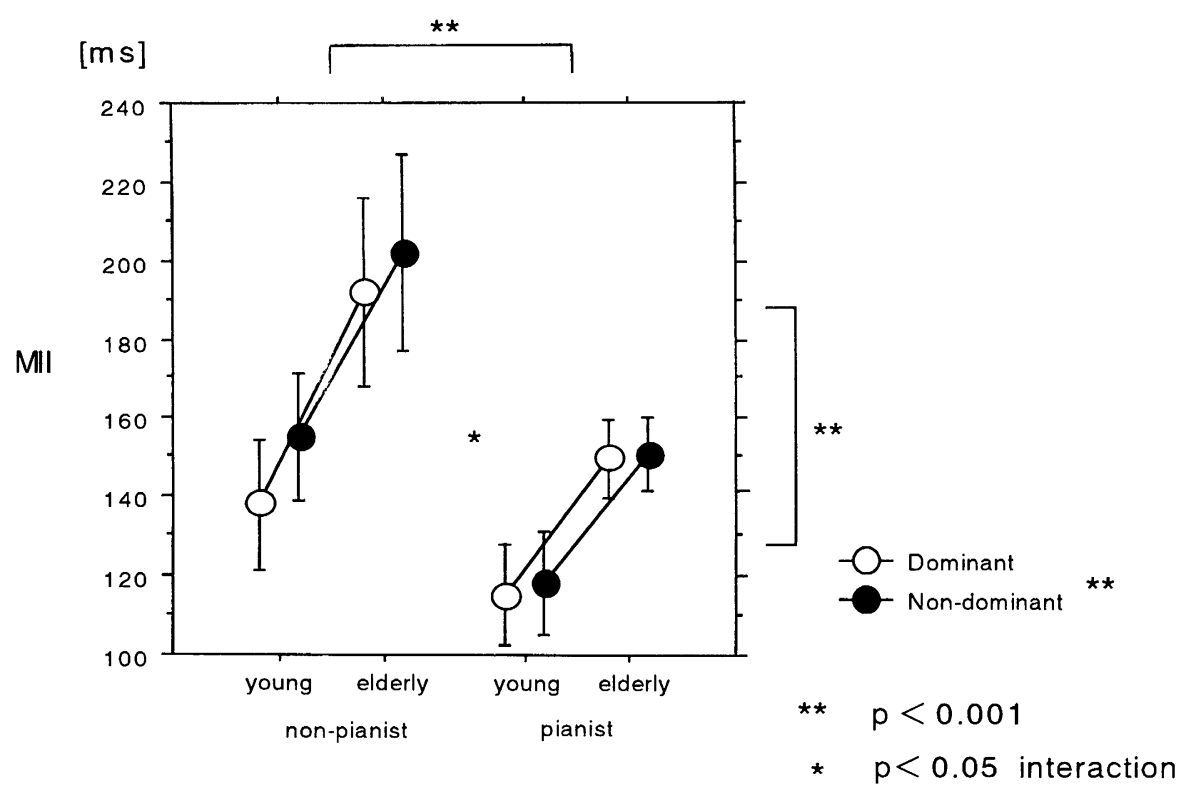

Figure 3 MII (Mean Intertap Interval) of female pianist and non-pianist groups. Explanation: see Figure 2.

In the ANOVA, all main effects were significant. Only the interaction between hand dominance versus expertise was significant. Using a post-hoc test (Tukey's HSD) to clarify the relationship between expertise and hand dominance, we found no significant difference between hands in pianists $(p<0.05)$. Pianists tapped more frequently than non-pianists and had balanced agility in both hands (Figure 3). Nevertheless, pianists experience a lowering of their frequency in both hands with aging, but it was not significantly less pronounced than in non-pianists.

\section{Discussion}

Repetitive tapping occurs as a result of a smooth on-off interaction between antagonist and agonist in the wrist and MP-joint. Thus, repetitive tapping frequency can be considered a good quantitative physical parameter of coordinated manual skills like piano playing or typing.

The new finding of this research is that rates of age-related decrease are similar in both dominant and non-dominant hands whether the subject be non-pianist or pianist. The effect of constant exercise in professional pianists, however, is to keep their finger movements faster than those of non-pianists. Nevertheless, even constant exercise cannot overcome an age-related decrease in agility. The most remarkable finding in professional pianists is that they exhibit equivalent dexterity between dominant and non-dominant hands both in young and elderly subjects.

These findings indicate that age-related regular deterioration in the speed of manual 
skill cannot be avoided, irrespective of the large amounts of daily training in both hands as in case of experienced pianists or greater daily exercise in the dominant hand of non-pianists.

We hypothesize that the effects of exercise and aging are independent phenomena in order to explain trends of manual dexterity in the present study. In this hypothesis, all individuals experience deterioration in agility due to aging. Higher and equivalent agility in the hands of pianists and in the dominant hand of non-pianists depends to a degree on habituation of hand use and the consequent motor programming in the brain from their early childhood to adolescence. Moderate to moderately high heritability also reported to effect manual agility (Boucheard and others, 1997). An age-related decrease in manual agility occurs because of deteriorating maximum conduction velocities in muscle contractions (Larsson and Karlsson, 1978) and neural contacts (Norris and others, 1953) as part of normal trends in aging. Effects of exercise or training have more to do with the controlling systems of the central nervous system (Spirduso, 1995). Morphological changes due to practice or training were anatomically examined in the brains of experimental animals where it was found that the neural plasticity for practice and learning remains even in the aged brain (Floeter and Greenough, 1979; Pysh and Weiss, 1979). We further hypothesize that the physiological causes cannot interact with each other. As a result, pianists cannot prevent senescent velocities in muscle contraction or peripheral neural contacts from occurring through daily training. Daily training of pianists might only maintain equal dexterity in both hands and their motor programs in central nerve system because of this neural plasticity.

Another possibility remains whereby aging and training interact with each other. Habitual physical activity and training, in general, tend to offset and slow the age-related decline in various motor performance (Boucheard and others, 1997). The common rate of the age-related decrease of manual agility in the present study is an apparent delay derived from an offset of training and aging effects. Daily training in pianists might maintain for longer superior physiological characteristics in nerves and muscles to postpone the natural effect of aging. However, this second hypothesis cannot account for the same tendency of age-related decrease between pianists and non-pianists as shown in the present experiment (Figure 3).

This study has focused on agility in simple manual coordination. There still remain unresolved questions to fully describe the nature of aging and impact of training effects on such a simple movement. Correlational studies are need for both longitudinal and physiological assessments of altered skills in both elderly persons and expert pianists.

\section{Acknowledgments}

Our thanks are due to Prof. T. Hosokawa, Prof. H. Nagasaki and Ms. E. Okuma for their continuous guidance and help, and to all the participants in the present study. This work was partly supported by grants from Japan Foundation for Aging and Health. 


\section{REFERENCES}

Bouchard C., Malina R.M., and Perusse L. (1997) Genetics of Fitness and Physical Performance. Human Kinetics, Champaign.

Fleishman E.A. and Quaintance M.K. (1984) Taxonomies of human performance. Academic Press, Orland.

Floeter M. and Greenough W.T. (1979) Cerebellar plasticity: modification of Purkinje cell structure by differential rearing in monkeys. Science, vol. 206, pp. 227-229.

Hashizumè K., Nagasaki H., Itoh H., Furuna T., Sugiura M., Kinugasa T., and Maruyama H. (1994) Age-related difficulty in rhythmic movement. Japanese Journal of Gerontology, vol. 31, pp. 360-365.

Kapandji I.A. (1974) Physiologie articulaire. Fascicule 1. Librairie Maloine S.A., Paris (in French).

Larsson L. and Karlsson J. (1978) Isometric and dynamic endurance as a function of age and skeletal muscle characteristics. Acta Physiologica Scandinavica, vol. 104, pp. 129-136.

Nagasaki H., Itoh H., Maruyama H., and Hashizume K. (1988) Characteristics difficulty in rhythmic movement with aging and its relation to Parkinsons' disease. Experimental Aging Research, vol. 14, pp. 171-176.

Norris A.H., Shock N.W., and Wagman I.H. (1953) Age changes in the maximum conduction velocity of motor fibers of human ulnar nerves. Journal of Applied Physiology, vol. 5, pp. 589-593.

Pysh J.J. and Weiss G.M. (1979) Exercise during development induces an increase in Purkinje cell dendritic tree size. Science, vol. 206, pp. 230-231.

Spirduso, W.W. (1995) Physical dimensions of aging. Human Kinetics, Champaign.

Handling editor: Saitou Naruya 